\title{
ON ENDO CURVATURE TENSOR OF A CONTACT METRIC MANIFOLD
}

\author{
MOHIT KUMAR DWIVEDI, JAE-BOK JUN AND MUKUT MANI TRIPATHI
}

\begin{abstract}
We prove that a $(k, \mu)$-manifold with vanishing Endo curvature tensor is a Sasakian manifold. We find a necessary and sufficient condition for a non-Sasakian $(k, \mu)$-manifold whose Endo curvature tensor $B^{e s}$ satisfies $B^{e s}(\xi, X) \cdot S=0$, where $S$ is the Ricci tensor. Using $\mathscr{D}$-homothetic deformation we obtain an example of an $N(k)$ contact metric manifold on which $B^{e s}(\xi, X) \cdot S \neq 0$.
\end{abstract}

\section{Introduction}

In [2], Blair, Koufogiorgos and Papantoniou introduced the class of contact metric manifolds $M$ with contact metric structures $(\varphi, \xi, \eta, g)$, in which the curvature tensor $R$ satisfies the equation

$$
R(X, Y) \xi=(k I+\mu h)(X \wedge Y) \xi, \quad X, Y \in T M,
$$

where $(k, \mu) \in \mathbb{R}^{2}, 2 h$ is the Lie derivative of $\varphi$ in the direction $\xi$ and

$$
(X \wedge Y) Z=g(Y, Z) X-g(X, Z) Y, \quad X, Y, Z \in T M
$$

A contact metric manifold belonging to this class is called a $(k, \mu)$-manifold. A $(k, \mu)$-manifold becomes a Sasakian manifold if $k=1$. Characteristic examples of non-Sasakian $(k, \mu)$-manifolds are the tangent sphere bundles of Riemannian manifolds of constant sectional curvature not equal to one and certain Lie groups.

On the other hand, Bochner introduced a Kähler analogue of the Weyl conformal curvature tensor, which is now well known as the Bochner curvature tensor [3]. By using the Boothby-Wang's fibration, Matsumoto and Chūman constructed C-Bochner curvature tensor [9] from the Bochner curvature tensor and studied in the context of Sasakian geometry. In a Sasakian manifold, the Ricci operator $Q$ commutes with the structure tensor $\varphi$, but in general $Q \varphi \neq \varphi Q$. Thus the definition of $C$-Bochner curvature tensor seems not to include all the non-Sasakian cases. Perhaps keeping this view in mind, Endo [6] defined a curvature tensor $B^{e s}$ on a contact metric manifold, which coincides with the $C$-Bochner curvature tensor when the manifold is Sasakian.

Received March 26, 2007.

2000 Mathematics Subject Classification. 53C25, 53D10.

Key words and phrases. Contact metric manifold, $(k, \mu)$-manifold, $N(k)$-contact metric manifold, Sasakian manifold, Endo curvature tensor, Einstein manifold, $\eta$-Einstein manifold. 
In this paper, we recognize the curvature tensor $B^{e s}$ as Endo curvature tensor. In Section 2, we give necessary details about contact metric manifolds and Sasakian manifolds. In Section 3, we prove that an $\xi$-Endo flat $(k, \mu)$-manifold is a Sasakian manifold. In particular, an Endo flat $(k, \mu)$-manifold is a Sasakian manifold. We also prove that if the Endo curvature tensor vanishes on the unit tangent sphere bundle $T_{1} M(c)$ of a manifold of constant curvature $c$, then $c=1$. In [13], [14] and [17], contact metric manifolds satisfying $R(X, \xi) \cdot S=0$ are studied. Motivated by these studies, in Section 4 , we prove that in a non-Sasakian $(k, \mu)$ manifold the Endo curvature tensor $B^{e s}$ satisfies $B^{e s}(\xi, X) \cdot S=0$ if and only if the manifold is 3-dimensional and flat. In last, we also give an example of an $N(k)$-contact metric manifold, on which $B^{e s}(\xi, X) \cdot S \neq 0$.

\section{Contact metric manifolds}

A $(2 n+1)$-dimensional differentiable manifold $M$ is called an almost contact manifold if there is an almost contact structure $(\varphi, \xi, \eta)$ consisting of a $(1,1)$ tensor field $\varphi$, a vector field $\xi$, and a 1-form $\eta$ satisfying

$$
\varphi^{2}=-I+\eta \otimes \xi, \quad \text { and (one of) } \quad \eta(\xi)=1, \quad \varphi \xi=0, \quad \eta \circ \varphi=0 .
$$

Let $g$ be a compatible Riemannian metric with $(\varphi, \xi, \eta)$, that is,

$$
g(X, Y)=g(\varphi X, \varphi Y)+\eta(X) \eta(Y)
$$

or equivalently,

$$
g(X, \varphi Y)=-g(\varphi X, Y) \quad \text { and } \quad g(X, \xi)=\eta(X)
$$

for all $X, Y \in T M$. Then, $M$ becomes an almost contact metric manifold equipped with an almost contact metric structure $(\varphi, \xi, \eta, g)$.

An almost contact metric structure becomes a contact metric structure if

$$
g(X, \varphi Y)=d \eta(X, Y), \quad X, Y \in T M .
$$

In a contact metric manifold, the $(1,1)$-tensor field $h$ is symmetric and satisfies

$$
h \xi=0, \quad h \varphi+\varphi h=0, \quad \operatorname{trace}(h)=\operatorname{trace}(\varphi h)=0 .
$$

A contact metric manifold $M$ is Sasakian if and only if

$$
R(X, Y) \xi=(X \wedge Y) \xi, \quad X, Y \in T M .
$$

The $(k, \mu)$-nullity distribution $N(k, \mu)$ ([2],[13]) of a contact metric manifold $M$ is defined by

$$
N(k, \mu): p \rightarrow N_{p}(k, \mu)=\left\{Z \in T_{p} M: R(X, Y) Z=(k I+\mu h)(X \wedge Y) Z\right\}
$$

for all $X, Y, Z \in T M$, where $(k, \mu) \in \mathbb{R}^{2}$. A contact metric manifold with $\xi \in N(k, \mu)$ is called a $(k, \mu)$-manifold. For a $(k, \mu)$-manifold, it follows that $h^{2}=(k-1) \varphi^{2}$. This class contains 
Sasakian manifolds for $k=1$.In fact, for a $(k, \mu)$-manifold, the conditions of being Sasakian manifold, $k=1$ and $h=0$ are all equivalent. If $\mu=0$, the $(k, \mu)$-nullity distribution $N(k, \mu)$ is reduced to the $k$-nullity distribution $N(k)$ [15]. If $\xi \in N(k)$, then we call a contact metric manifold $M$ an $N(k)$-contact metric manifold. For more details we refer to [1].

\section{3. $(k, \mu)$-manifolds with vanishing Endo curvature tensor}

In [6], Endo defined a tensor in a $(2 n+1)$-dimensional contact metric manifold $M$ as follows:

$$
\begin{aligned}
B^{e s}(X, Y)=R(X, Y)+h \varphi X \wedge h \varphi Y \\
+\frac{1}{4(n+2)}\{Q Y \wedge X-\varphi Q \varphi Y \wedge X \\
\quad+\frac{1}{2}\{\eta(Y) Q \xi \wedge X+\eta(Q Y) \xi \wedge X\} \\
\quad-Q X \wedge Y+\varphi Q \varphi X \wedge Y \\
\quad-\frac{1}{2}\{\eta(X) Q \xi \wedge Y+\eta(Q X) \xi \wedge Y\} \\
\quad+Q \varphi Y \wedge \varphi X+\varphi Q Y \wedge \varphi X \\
\quad-Q \varphi X \wedge \varphi Y-\varphi Q X \wedge \varphi Y \\
\quad+2 g(Q \varphi X, Y) \varphi+2 g(\varphi Q X, Y) \varphi \\
\quad+2 g(\varphi X, Y) \varphi Q+2 g(\varphi X, Y) Q \varphi \\
\quad-\eta(X) Q Y \wedge \xi+\eta(X)(\varphi Q \varphi Y) \wedge \xi \\
\quad+\eta(Y) Q X \wedge \xi-\eta(Y)(\varphi Q \varphi X) \wedge \xi\} \\
-\frac{r+4 n^{2}+6 n}{4(n+1)(n+2)}\{\varphi Y \wedge \varphi X+2 g(\varphi X, Y) \varphi\} \\
-\frac{r-6 n-8}{4(n+1)(n+2)} Y \wedge X \\
+\frac{r+2 n}{4(n+1)(n+2)}\{\eta(Y) \xi \wedge X+\eta(X) Y \wedge \xi\} \\
-\frac{1}{4(n+1)(n+2)} \operatorname{trace}\left(h^{2}\right)\{\varphi Y \wedge \varphi X+2 g(\varphi X, Y) \varphi \\
\quad+Y \wedge X+\eta(Y) X \wedge \xi+\eta(X) \xi \wedge Y\},
\end{aligned}
$$

where $X, Y \in T M, Q$ is the Ricci operator and $r$ is the scalar curvature. The tensor $B^{e s}$ will be called the Endo curvature tensor.

In a $(2 n+1)$-dimensional $(k, \mu)$-manifold $M,(1.1)$ is equivalent to

$$
R(\xi, X)=\xi \wedge(k I+\mu h) X=-R(X, \xi), \quad X \in T M .
$$

From (3.1), (1.1) and (3.2), it follows that

$$
B^{e s}(X, Y) \xi=\left(\frac{2(k-1)}{n+2} I+\mu h\right)(X \wedge Y) \xi,
$$




$$
B^{e s}(\xi, X)=\xi \wedge\left(\frac{2(k-1)}{n+2} I+\mu h\right) X=-B^{e s}(X, \xi)
$$

for all $X, Y \in T M$. Consequently, we have

$$
\begin{gathered}
B^{e s}(\xi, X) \xi=\frac{2(k-1)}{n+2}(\eta(X) \xi-X)-\mu h X=-B^{e s}(X, \xi) \xi . \\
\eta\left(B^{e s}(X, Y) \xi\right)=0, \\
\eta\left(B^{e s}(\xi, X) Y\right)=\frac{2(k-1)}{n+2}(g(X, Y)-\eta(X) \eta(Y))+\mu g(h X, Y) .
\end{gathered}
$$

Now, we need the following definition.

Definition 3.1. A $(k, \mu)$-manifold $M$ is said to be $\xi$-Endo flat if

$$
B^{e s}(X, Y) \xi=0, \quad X, Y \in T M .
$$

In particular, if $B^{e s}=0$ then $M$ will be said to be Endo flat.

Now, we prove the following

Theorem 3.2. An $\xi$-Endo flat $(k, \mu)$-manifold is a Sasakian manifold.

Proof. Let $M^{2 n+1}$ be an $\xi$-Endo flat $(k, \mu)$-manifold. Then in view of (3.5), we get

$$
0=\frac{2(k-1)}{n+2}(\eta(X) \xi-X)-\mu h X,
$$

which, in view of $h^{2}=(k-1) \varphi^{2}$, implies that

$$
\frac{(n+2) \mu}{2} h=h^{2} .
$$

Taking the trace of (3.8), we obtain

$$
0=\operatorname{trace}\left(h^{2}\right)=2 n(1-k) .
$$

From (3.9), we have $k=1$. Thus, $M^{2 n+1}$ becomes Sasakian.

Corollary 3.3. An Endo flat $(k, \mu)$-manifold is a Sasakian manifold.

A contact metric manifold $M$ is said to be $\eta$-Einstein [10] if the Ricci tensor $S$ satisfies

$$
S=a g+b \eta \otimes \eta,
$$

where $a$ and $b$ are smooth functions on the manifold. In particular, if $b=0$ then $M$ is an Einstein manifold. 
The Endo curvature tensor reduces to $C$-Bochner curvature tensor in a Sasakian manifold. A number of results for Sasakian manifolds with vanishing $C$-Bochner curvature tensor can be found in [5], [7], [8], [11], [12] etc.. Here, we list some known results for Sasakian manifolds with vanishing $C$-Bochner curvature tensor in the following two Theorems.

Theorem 3.4. Let $M^{2 n+1}$ be a Sasakian manifold with vanishing C-Bochner curvature tensor. Then the following statements are true:

1. ([11]) If the scalar curvature $r$ is constant and the Ricci tensor is positive semi-definite, then $M^{2 n+1}$ is $\eta$-Einstein.

2. (Theorem 1, [12]) If the scalar curvature $r$ is constant and the square of the length of the Ricci tensor is less than $r^{2} / 2 n$, then $M^{2 n+1}$ is $\eta$-Einstein.

3. (Theorem 1, [7]) If (i) $n \geq 3$, (ii) the scalar curvature $r$ is constant and (iii) the square of the length of the $\eta$-Einstein tensor

$$
S_{i j}=\left(\frac{r}{2 n}-1\right) g_{i j}-\left(\frac{r}{2 n}-2 n-1\right) \eta_{i} \eta_{j}
$$

is less than $\frac{(n-1)(n+2)^{2}(r+2 n)^{2}}{2 n(n+1)^{2}(n-2)^{2}}$; then $M^{2 n+1}$ is a Sasakian space form.

4. (Theorem 2, [7]) If $n=2$ and the scalar curvature is a constant $r \neq-4$, then $M^{2 n+1}$ is a Sasakian space form.

5. (Theorem B, [5]) If (i) $n \geq 2$, (ii) the scalar curvature is constant and (iii) the Ricci tensor is positive semi-definite; then $M^{2 n+1}$ is a Sasakian space form.

THeorem 3.5. Let $M^{2 n+1}$ be a compact Sasakian manifold with vanishing C-Bochner curvature tensor. Then the following statements are true:

1. (Theorem 2, [12]) If the square of the length of the Ricci tensor is constant and less than $r^{2} / 2 n$, where $r$ is the scalar curvature; then $M^{2 n+1}$ is $\eta$-Einstein.

2. (Theorem 4.1, [9]) If (i) $n \geq 2$, (ii) the scalar curvature is constant and (iii) the smallest eigenvalue of the Ricci tensor is greater than-2, then $M^{2 n+1}$ is $\eta$-Einstein.

3. (Theorem C, [5]) If (i) $n \geq 3$, (ii) the length of the Ricci tensor is constant and (iii) the length of the $\eta$-Einstein tensor

$$
S_{i j}=\left(\frac{r}{2 n}-1\right) g_{i j}-\left(\frac{r}{2 n}-2 n-1\right) \eta_{i} \eta_{j}
$$

is less than $\frac{\sqrt{2}(r-n+1)}{\sqrt{(n-1)(n-3)}}$, then $M^{2 n+1}$ is a Sasakian space form. 
Remark 3.6. In view of Corollary 3.3, the consequences of Theorem 3.4 and Theorem 3.5 are still true if Sasakian manifolds with vanishing $C$-Bochner curvature tensor are replaced by Endo-flat $(k, \mu)$-manifolds.

The unit tangent sphere bundle $T_{1} M$ equipped with the standard contact metric structure is a $(k, \mu)$-manifold if and only if the base manifold $M$ is of constant curvature $c$ with $k=$ $c(2-c)$ and $\mu=-2 c$ [2]. In case of $c \neq 1$, the unit tangent sphere bundle is non-Sasakian [16]. Then, applying Theorem 3.2 to $T_{1} M(c)$, we have

Corollary 3.7. If the unit tangent sphere bundle $T_{1} M(c)$ of a space of constant curvature $c$ is Endo flat then $c=1$.

\section{4. $(k, \mu)$-manifolds satisfying $B^{e s}(\xi, X) \cdot S=0$}

In a non-Sasakian $(k, \mu)$-manifold $M^{2 n+1}$ the Ricci tensor $S$ is given by [2]

$$
\begin{aligned}
S(X, Y)= & (2(n-1)-n \mu) g(X, Y)+(2(n-1)+\mu) g(h X, Y) \\
& +(2(1-n)+n(2 k+\mu)) \eta(X) \eta(Y)
\end{aligned}
$$

for all $X, Y \in T M$.

We also recall the following result for later use.

Theorem 4.1. (Theorem 1.2, [18]) A non-Sasakian Einstein $(k, \mu)$-manifold is flat and 3 dimensional.

Now we prove the main result of this section as follows:

Theorem 4.2. Let $M$ be a non-Sasakian $(k, \mu)$-manifold. Then the Endo curvature tensor $B^{e s}$ satisfies $B^{e s}(\xi, X) \cdot S=0$ if and only if $M$ is flat and 3 -dimensional.

Proof. Let $M^{2 n+1}$ be a non-Sasakian $(k, \mu)$-manifold. Then the condition $B^{e s}(\xi, X) \cdot S=0$ gives

$$
S\left(B^{e s}(\xi, X) Y, \xi\right)+S\left(Y, B^{e s}(\xi, X) \xi\right)=0 .
$$

In view of $Q \xi=2 n k \xi$, we get

$$
S(X, \xi)=2 n k \eta(X)
$$

which implies that

$$
S\left(B^{e s}(\xi, X) Y, \xi\right)=2 n k \eta\left(B^{e s}(\xi, X) Y\right) .
$$

Using (3.7) in (4.4), we obtain

$$
\begin{aligned}
S\left(B^{e s}(\xi, X) Y, \xi\right)= & 2 n k \mu g(h X, Y) \\
& +\frac{4 n k(k-1)}{n+2}(g(X, Y)-\eta(X) \eta(Y)) .
\end{aligned}
$$


In view of (3.5) and (4.3) we have

$$
\begin{aligned}
S\left(B^{e s}(\xi, X) \xi, Y\right)= & \frac{4 n k(k-1)}{n+2} \eta(X) \eta(Y) \\
& -\frac{2(k-1)}{n+2} S(X, Y)-\mu S(h X, Y) .
\end{aligned}
$$

From (4.2), (4.5) and (4.6), we have

$$
\begin{aligned}
\frac{2(k-1)}{n+2} S(X, Y)= & \frac{4 n k(k-1)}{n+2} g(X, Y) \\
& +2 n \mu g(h X, Y)-\mu S(h X, Y) .
\end{aligned}
$$

From (4.1) we have

$$
\begin{aligned}
S(h X, Y)= & (2(n-1)-n \mu) g(h X, Y) \\
& -(k-1)(2(n-1)+\mu) g(X, Y) \\
& +(k-1)(2(n-1)+\mu) \eta(X) \eta(Y),
\end{aligned}
$$

where we have used $\eta \circ h=0, h^{2}=(k-1) \varphi^{2}$ and (2.2). In view of (4.1), (4.7) and (4.8) we obtain (3.10) with

$$
\begin{aligned}
a= & \frac{(2(n-1)+\mu)(k-1)(4 n k+(n+2)(2(n-1)+\mu) \mu)}{2(k-1)(2(n-1)+\mu)-\mu(n+2)(2+n \mu)} \\
& -\frac{\mu(n+2)(2+n \mu)(2(n-1)-n \mu)}{2(k-1)(2(n-1)+\mu)-\mu(n+2)(2+n \mu)},
\end{aligned}
$$

and

$$
b=-\frac{\mu(n+2)\left((k-1)(2(n-1)+\mu)^{2}+(2+n \mu)(n(2 k+\mu)-2(n-1))\right)}{2(k-1)(2(n-1)+\mu)-\mu(2+n \mu)(n+2)} .
$$

Thus we see that $M^{2 n+1}$ becomes $\eta$-Einstein. From (4.1) and (3.10), we see that a nonSasakian $(k, \mu)$-manifold $M^{2 n+1}$ is $\eta$-Einstein if and only if

$$
\mu=-2(n-1) .
$$

Using (4.11) in (4.1) we get

$$
S=2\left(n^{2}-1\right) g-2\left(n^{2}-n k-1\right) \eta \otimes \eta .
$$

It can be noted that in view of (4.9), (4.10) and (4.11), equation (3.10) also yields (4.12). From (4.12), it follows that

$$
r=2 n(k+2(n-1)(n+1)) .
$$

Using (4.11) in (4.8) we get

$$
S(h X, Y)=2\left(n^{2}-1\right) g(h X, Y) .
$$


Using (4.11) and (4.14) in (4.7) and contracting the resulting equation we obtain

$$
0=(k-1)(2 n k(2 n+1)-r),
$$

where trace $(h)=0$ is used. In view of (4.13), (4.15) and $k<1$, we obtain

$$
n^{2}-n k-1=0 .
$$

Using (4.16) in (4.12) we see that $M^{2 n+1}$ is Einstein and consequently in view of Theorem 4.1, $M^{2 n+1}$ becomes flat and 3-dimensional.

The converse is straightforward.

In last, we give an example of an $N(k)$-contact metric manifold, on which $B^{e s}(\xi, X) \cdot S \neq 0$.

First, we recall the notion of a $\mathscr{D}_{a}$-homothetic deformation. For a given contact metric structure $(\varphi, \xi, \eta, g)$, this is the structure defined by

$$
\bar{\eta}=a \eta, \quad \bar{\xi}=\frac{1}{a} \xi, \quad \bar{\varphi}=\varphi, \quad \bar{g}=a g+a(a-1) \eta \otimes \eta,
$$

where $a$ is a positive constant. While such a change preserves the state of being contact metric or Sasakian, it destroys a condition like $R(X, Y) \xi=0$ or $R(X, Y) \xi=k(\eta(Y) X-\eta(X) Y)$. However the form of the $(k, \mu)$-nullity condition, that is

$$
R(X, Y) \xi=(k I+\mu h)(\eta(Y) X-\eta(X) Y), \quad X, Y \in T M
$$

for $k, \mu$ being real constants, is preserved under a $\mathscr{D}_{a}$-homothetic deformation with

$$
\bar{k}=\frac{k+a^{2}-1}{a^{2}}, \quad \bar{\mu}=\frac{\mu+2 a-2}{a} .
$$

Given a non-Sasakian $(k, \mu)$-manifold $M$, Boeckx [4] introduced an invariant $I_{M}=(1-\mu / 2) / \sqrt{1-k}$ and showed that two non-Sasakian $(k, \mu)$-manifolds $M_{1}$ and $M_{2}$ are locally isometric as contact metric manifolds up to a $\mathscr{D}_{a}$-homothetic deformation if and only if $I_{M_{1}}=I_{M_{2}}$.

Now, we present the following

Example 4.3. Consider the unit tangent sphere bundle of a 4-dimensional manifold of constant curvature $(3+2 \sqrt{2})$. After a $\mathscr{D}_{2(2+\sqrt{2})}$-homothetic deformation we get an $N(1 / 2)$ contact metric manifold, on which $B^{e s}(\xi, X) \cdot S \neq 0$.

\section{Acknowledgement}

The first author is grateful to University Grants Commission, New Delhi for financial support in the form of Junior Research Fellowship. Authors are thankful to the referee for suggestions for improvement of the paper. The second author is thankful to Kookmin University for partial support (KMU 2008). 


\section{REFERENCES}

\section{References}

[1] D. E. Blair, Riemannian geometry of contact and symplectic manifolds, Progress in Mathematics, 203. Birkhauser Boston, Inc., Boston, MA, 2002.

[2] D. E. Blair, T. Koufogiorgos and B. J. Papantoniou, Contact metric manifolds satisfying a nullity condition, Israel J. Math. 91(1995), 189-214.

[3] S. Bochner, Curvature and Betti numbers, II Ann. of Math. 50(1949), 77-93.

[4] E. Boeckx, A full classification of contact metric $(k, \mu)$-spaces, Illinois J. Math. 44(2000), 212-219.

[5] E.-S. Choi, U.-H. Ki and K. Takano, Sasakian manifolds with vanishing C-Bochner curvature tensor, Acta Math. Hungarica 66(1995), 327-336.

[6] H. Endo, On certain tensor fields on contact metric manifolds II, Publ. Math. Debrecen 44(1994), $157-166$.

[7] I. Hasegawa and T. Nakane, On Sasakian manifolds with vanishing contact Bochner curvature tensor, Hokkaido Math. J. 9(1980), 184-189.

[8] U.-H. Ki and H. S. Kim, Sasakian manifolds whose C-Bochner curvature tensor vanishes, Tensor (N.S.) 49(1990), 32-39.

[9] M. Matsumoto, G. Chūman, On the C-Bochner curvature tensor, TRU Math. 5(1969), 21-30.

[10] M. Okumura, Some remarks on space with a certain contact structure, Tôhoku Math. J. 14(1962), 135-145.

[11] J. S. Pak, A note on Sasakian manifolds with vanishing C-Bochner curvature tensor, Kodai Math. Sem. Rep. 28(1976), 19-27.

[12] J. S. Pak, On Sasakian manifolds with vanishing C-Bochner curvature tensor, Kyungpook Math. J. 17(1977), 123-133.

[13] B. J. Papantoniou, Contact Riemannian manifolds satisfying $R(\xi, X) \cdot R=0$ and $\xi \in(k, \mu)$-nullity distribution, Yokohama Math. J. 40(1993), 149-161.

[14] D. Perrone, Contact Riemannian manifolds satisfying $R(X, \xi) \cdot R=0$, Yokohama Math. J. 39(1992), 141-149.

[15] S. Tanno, Ricci curvatures of contact Riemannian manifolds, Tôhoku Math. J. 40(1988), 441-448.

[16] Y. Tashiro, On contact structures of tangent sphere bundles, Tôhoku Math. J. 21(1969), 117-143.

[17] M. M. Tripathi, Classification of $\xi$-Ricci-semisymmetric $(\kappa, \mu)$-manifolds, Balkan J. Geom. Appl. 11(2006), 135-143.

[18] M. M. Tripathi and J.-S. Kim, On the concircular curvature tensor of a $(\kappa, \mu)$-manifold, Balkan J. Geom. Appl. 9(2004), 114-124.

Department of Mathematics and Astronomy, Lucknow University, Lucknow 226 007, India.

E-mail: dwivedimohit@yahoo.com

Department of Mathematics, Kookmin University, Seoul, South Korea.

E-mail: jbjun@kookmin.ac.kr

Department of Mathematics and Astronomy, Lucknow University, Lucknow 226 007, India. Present Address: Department of Mathematics, Banaras Hindu University, Varanasi 221 005, India.

E-mail: mmtripathi66@yahoo.com 